\title{
Resistance and Gross-resistance of Escherichia coli Mutants to Antitumour Agent Mitomycin G
}

\author{
By J. GREENBERG,* J. D. MANDELL* AND PEARL L. WOODY* \\ Stanford Research Institute, Menlo Park, California, U.S.A.
}

(Received 27 April 1961)

\begin{abstract}
SUMMARY
The cross-resistance patterns are described for 96 mutants selected in one step from Escherichia coli strain S for resistance to Mitomycin $\mathbf{C}$. The test agents used were ultraviolet radiation, seven radiomimetic and two non-radiomimetic compounds. Seven different types of mutants could be selected in one step from the parent. Five of these were radioresistant; two were chemoresistant. Of the radioresistant types two were identical with types previously isolated using other radiomimetic agents for selection; three of the types were new. One of the new types was reactivated by plating medium following ultraviolet irradiation differently from all other radioresistant mutants of $\boldsymbol{E}$. coli S. One of the chemoresistant types was resistant only to Mitomycin C; the other displayed a low degree of cross-resistance to nitronitrosoguanidines and to penicillin. Also described are the cross-resistance patterns of mutants selected in five consecutive steps for increasing resistance to Mitomycin $\mathrm{C}$. Beginning with a first step radioresistant mutant it was possible to select four additional steps, up to 325-fold, in resistance to Mitomycin C. One of these steps appeared to be a shift from one radioresistant type to another.
\end{abstract}

\section{INTRODUCTION}

This paper describes the cross-resistance patterns of mutants of Escherichia coli strain S, selected for resistance to Mitomycin C, an antibiotic with antitumour activity (Usubuchi et al. 1957). Mitomycin $\mathrm{C}$ has radiomimetic properties : in bacteria it is a mutagen (Szybalski, 1958) and like radiation it selectively inhibits the synthesis of deoxyribonucleic acid (Shiba, Terawaki, Taguchi \& Kawamata, 1959). Mutants of $\boldsymbol{E}$. coli $\mathrm{S}$ selected for resistance to other radiomimetic agents were cross-resistant to Mitomycin C (Mandell, Woody \& Greenberg, 1961; Woody, Mandell \& Greenberg, 1961 ; Greenberg, Mandell \& Woody, 1961). Furthermore, Oboshi (1959) found that tumours selected for resistance to nitrogen mustard N-oxide (nitromin) were also resistant to Mitomycin C.

In earlier work, 13 first-step mutants of Escherichia coli $\mathrm{S}$ were selected for resistance to 1-methyl-3-nitro-1-nitrosoguanidine (Mandell et al. 1961), to nitrogen mustard and to nitromin (Woody et al. 1961), to azaserine, to 1-chloropropyl- and 1-chloroethyl-3-nitro-1-nitrosoguanidine (Greeenberg et al. 1961). Each mutant was characterized by its cross-resistance pattern, i.e. its degree of resistance to ultraviolet radiation, seven radiomimetic and two non-radiomimetic agents (penicillin

* Present address: Palo Alto Medical Research Foundation, 860 Bryant Street, Palo Alto, California, U.S.A. 
and 6-diazo-5-oxo-L-norleucine). The cross-resistance patterns of the 13 mutants are shown in Table 1 . Three of the mutants (S/Ni $1 a, \mathrm{~S} / \mathrm{As} 1 a$ and S/Cp 1 $b$ ) were significantly resistant only to the selecting agent or to closely related chemicals. Such mutants have been termed 'chemoresistant' to indicate that resistance is to a specific chemical structure and not to the class of radiomimetic agents. The other ten mutants were resistant to all radiomimetic agents and to ultraviolet radiation but not to the non-radiomimetic agents. These have been called 'radioresistant' mutants (with the limitation that the effect of ionizing radiations on these mutants has not yet been tested). Four different cross-resistance patterns were observed among the 10 radioresistant mutants; these have been designated $R_{1}, R_{2}, R_{3}$ and $R_{4}$. $R_{1}$ and $R_{2}$ patterns were observed once each; $R_{3}$ and $R_{4}$ patterns occurred in mutants selected for resistance to three and five, respectively, different chemical agents. All Table 1. Cross-resistance among first step mutants resistant to
radiomimetic compounds

$\overbrace{\text { MC* }}^{\text {NG } \underset{\text { Minimum inhibitory concentration for Escherichia coli } \text { S } \dagger}{\text { CE }} \text { NM } \underset{\text { NI }}{\text { AS }} \text { DON }}$ PN

\begin{tabular}{|c|c|c|c|c|c|c|c|c|c|c|c|c|}
\hline & $\begin{array}{l}\text { Radio- } \\
\text { resistant }\end{array}$ & $\begin{array}{c}0.038 \\
\pm \\
0 . \overline{005}\end{array}$ & $\begin{array}{c}0.038 \\
\pm \\
0.006\end{array}$ & $\begin{array}{c}0.018 \\
\pm \\
0.002\end{array}$ & $\begin{array}{c}0.021 \\
\pm \\
0.003 \\
\end{array}$ & $\begin{array}{l}13 \\
\pm \\
2\end{array}$ & $\begin{array}{l}18 \\
\pm \\
3\end{array}$ & $\begin{array}{c}0.004 \\
\pm \\
0.001\end{array}$ & $\begin{array}{c}0.04 \\
\pm \\
0.02\end{array}$ & $\begin{array}{c}2 \cdot 2 \\
\pm \\
0 \cdot 2\end{array}$ & \multicolumn{2}{|c|}{$\begin{array}{c}22 \\
\text { Plated on }\end{array}$} \\
\hline $\begin{array}{l}\text { Bacterial } \\
\text { strain }\end{array}$ & $\begin{array}{l}\text { designa- } \\
\text { tion }\end{array}$ & \multicolumn{9}{|c|}{ Resistance factor } & $T 7 \cdot 0$ & M-9 \\
\hline S/Ni $1 \mathrm{a} \S$ & 一 & 1 & 1.2 & 1 & 1 & 1.2 & 60 & 1 & 1 & 1 & 1 & 6 \\
\hline S/As $1 \mathrm{a}$ & - & 1 & 0.7 & 1 & 1 & 1 & 1 & 16 & 1 & 1 & $\mathbf{1}$ & - \\
\hline $\mathrm{S} / \mathrm{Cp} 1 \mathrm{~b}$ & - & $1 \cdot 2$ & 12 & 9 & $9 \cdot 5$ & $0 \cdot 18$ & 0.22 & $1 \cdot 1$ & $\mathbf{I}$ & 1 & 1 & - \\
\hline $\mathrm{S} / \mathrm{Cp} 1 \mathrm{a}$ & $\mathbf{R}_{1}$ & $2 \cdot 7$ & $2 \cdot 0$ & $2 \cdot 3$ & $1 \cdot 6$ & $\mathbf{2} \cdot \boldsymbol{9}$ & 60 & $4 \cdot 0$ & 1 & 1 & 15 & 15 \\
\hline $\begin{array}{l}\text { S/Ni } 1 \text { b } \\
\text { S/Nm 1a }\end{array}$ & $\mathbf{R}_{2}$ & $5 \cdot 8$ & 19 & $6 \cdot 5$ & $6 \cdot 2$ & $6 \cdot 2$ & 60 & $6 \cdot 8$ & 1 & $0 \cdot 34$ & 15 & 15 \\
\hline $\begin{array}{l}\text { S/Ni 1c } \\
\text { S/As 1 b } \\
\text { S/Ng 1 a } \\
\text { S/Nm 1 b }\end{array}$ & $\mathbf{R}_{\mathbf{3}}$ & 9 & 28 & 9 & $9 \cdot 5$ & 10 & 60 & 13 & 1 & 1 & 15 & 15 \\
\hline $\left.\begin{array}{l}\text { S/As 1c } \\
\text { S/Ce 1a } \\
\text { S/Cp 1c }\end{array}\right\}$ & $\mathbf{R}_{4}$ & 22 & 45 & 26 & 24 & 20 & 60 & 16 & 1 & 1 & 15 & 15 \\
\hline
\end{tabular}

* The following abbreviations will be used: Mitomycin C, MC; 1-methyl-3-nitro-1-nitrosoguanidine, NG; 1-chlorethyl-3-nitro-1-nitrosoguanidine, CE; 1-chloropropyl-3-nitro-nitrosoguanidine, CP; nitrogen mustard, NM ; nitromin, NI; azaserine, AS; 6-diazo-5-oxo-L-norleucine, DON; penicillin, PN; ultraviolet light, u.v.

$\dagger \mu \mathrm{g} . / \mathrm{ml}$. estimated from gradient plates (Szybalski \& Bryson, 1952) except the u.v. value is expressed in ergs $\mathrm{mm} .^{-2} /$ hit based on calibration of the u.v. source with T2 phage (Latarjet $e t$ al. 1953). 'Hit' is defined as - In (fraction of survivors). The survivors were plated on tryptone agar pH 7.0 and M-9 minimal agar pH 6.8; the fold-increase, an average of several determinations, represents a comparison with the survival of the parent strain (plated on tryptone agar $\mathrm{pH} 7 \cdot 0$ ) set at unity.

$\ddagger$ The figures in these columns represent the factor of resistance (fold-increase) compared with the parent strain set at unity.

$\$$ Resistance to specific agents is conventionally represented by a bar and the abbreviation of the agent to which the strain was first found resistant. The first number following the bar denotes the selective isolation step to resistance; subsequent letters and numbers identify particular mutants and their families. 
the radioresistant mutants exhibited an identical degree of resistance to ultraviolet light, unaffected by post-irradiation growth on defined or complex media. This is in contrast to $E$. coli B (Roberts \& Aldous, 1949) and its radioresistant mutant, B/r (Alper \& Gillies, 1960; Hill \& Simson, 1961) and to $E$. coli S (Woody et al. 1961) whose degree of resistance to ultraviolet radiation can be altered by the postirradiation growth medium. All the radioresistant mutants were equally resistant to nitromin, but differed in their degree of resistance to the other six radiomimetic agents. The $R_{1}$ mutant was least resistant to these agents, the $R_{4}$ mutants were most resistant, while $R_{2}$ and $R_{3}$ mutants were intermediate in their resistance. If only ultraviolet radiation and nitromin had been used to characterize these mutants, they would all have appeared identical. However, by using nine test compounds, four different cross-resistance patterns were observed, indicating four different types of radioresistant mutant.

The results of an analysis of $\mathbf{9 6}$ first-step and four higher-step mutants show that when Mitomycin $C$ is used as the selecting agent: (1) radioresistant mutants of the classes $R_{3}$ and $R_{4}$ occurred frequently, while representatives of the classes $R_{1}$ and $R_{2}$ were not isolated; (2) three radioresistant mutants with hitherto undetected crossresistance patterns were isolated, including one whose resistance to ultraviolet radiation could be altered by the nature of the post-irradiation growth medium; (3) two types of chemoresistant mutants were isolated-one resistant only to Mitomycin $\mathbf{C}$, the other with a low degree of cross-resistance to nitrosoguanidines and to penicillin and under certain circumstances to ultraviolet radiation; (4) it was possible to select five consecutive steps of increasing resistance, up to 325-fold, to Mitomycin C; (5) the second step conferred increased resistance to Mitomycin $\mathrm{C}$ without otherwise effecting the cross-resistance pattern of the first-step radioresistant parent; (6) an $\mathbf{R}_{\mathbf{4}}$ type mutant was derived in a single selective step from a type $R_{3}$ parent.

\section{METHODS}

Bacterial strains. Escherichia coli strain S, obtained from Dr A. D. Hershey, was the parent strain. The characteristics of the resistant mutants used as reference strains are given in the appropriate tables. Their derivation is described in earlier papers (Mandell et al. 1961; Woody et al. 1961).

Compounds. The chemicals used were: 1-methyl-3-nitro-1-nitrosoguanidine, purchased from the Aldrich Chemical Co., Milwaukee, Wisc., and recrystallized from ethanol; 1-chloropropyl- and 1-chlorethyl-3-nitro-1-nitrosoguanidine kindly furnished by Dr B. R. Baker, Stanford Research Institute, Menlo Park, California; azaserine, 6-diazo-5-oxo-L-norleucine, Mitomycin $\mathrm{C}$ and nitromin, supplied by the Cancer Chemotherapy National Service Center, Bethesda, Md.; and nitrogen mustard, a gift from Merck Sharp and Dohme, Rahway, N.J. All compounds were prepared in sterile distilled water immediately before use.

Media. The media used contained per litre of distilled water:

Tryptone agar. Tryptone, 10 g.; glucose, 1 g.; sodium citrate, 2.0 g.; sodium chloride, 8 g. and agar, 12 g. (BBL, Baltimore Biological Lab., Inc.); adjusted to $\mathrm{pH} 7 \cdot 0$ with sodium hydroxide or 5.5 with hydrochloric acid.

$\boldsymbol{M}$-9 agar. Dibasic sodium phosphate, 5.8 g.; monobasic potassium phosphate, 3.0 g.; ammonium chloride, 1.0 g.; sodium chloride, 0.5 g.; glucose, 2.0 g.; mag- 
nesium sulphate $\left(7 \mathrm{H}_{2} \mathrm{O}\right), \mathbf{2 5 0} \mathrm{mg}$; calcium chloride, $14 \mathrm{mg}$.; $1 \%$ gelatin solution, $10 \mathrm{ml}$; agar, 8.0 g. (Ionagar, Oxo Ltd., London).

Peptone broth. Peptone, 10 g.; beef extract, 3 g.; glucose, 1 g.; sodium chloride, 5 g.

Diluting fluid. Peptone, 2 g.; sodium chloride, 6 g.; magnesium sulphate $\left(7 \mathrm{H}_{2} \mathrm{O}\right)$, $0.5 \mathrm{~g}$.

Phosphate-buffered saline was $1 \%$ sodium chloride in 0.2 m-phosphate buffer, pH 6.8. Tryptone glucose extract agar was a commercial (Difco) preparation, on which cultures were preserved after isolation and identification.

Isolation of resistant mutants. Resistant mutants were isolated from plates of tryptone agar ( $\mathrm{pH} \mathrm{5.5)}$ ) containing graded doses over a 100-fold range of Mitomycin $\mathrm{C}$ and spread with $3.5 \times 10^{7}$ organisms of the appropriate parent, growing logarithmically in peptone broth.

Measurement of resistance to chemical agents. The methods used to measure the degree of resistance to chemical agents have been given in detail elsewhere (Mandell et al. 1961). All isolated clones were grown overnight in peptone broth at $37^{\circ}$, adjusted turbidimetrically to a population density of about $3.5 \times 10^{8} / \mathrm{ml}$, and streaked on gradient plates, according to the method of Szybalski \& Bryson (1952). Gradient plates were made with tryptone agar ( $\mathrm{pH}$ 5.5) except that M-9 agar was used in tests involving azaserine, 6-diazo-5-oxo-L-norleucine and penicillin. The minimum inhibitory concentration (MIC) was determined as follows:

$\frac{\text { length of solid growth }}{\text { total length of } \text { streak }} \times$ maximum concentration of test compound $(\mu \mathrm{g} . / \mathrm{ml}$.$) .$

Sensitivity to ultraviolet radiation. The ultraviolet radiation source was a single $15 \mathrm{~W}$. General Electric germicidal lamp with a maximum output at $2537 \AA$., calibrated with bacteriophage T2 according to the method of Latarjet, Morenne \& Berger (1953). Cultures grown overnight in peptone broth were washed twice with phosphate buffered saline, diluted to about $2 \times 10^{6}$ bacteria $/ \mathrm{ml}$. in cold buffered saline (pH 6.8) and exposed in $50 \mathrm{~mm}$. Petri dishes containing $1 \mathrm{ml}$. of bacterial suspension. Exposures were made at a distance of $51.5 \mathrm{~cm}$. from the ultraviolet radiation source. The dish was agitated gently by hand throughout exposure. Appropriate dilutions in cold diluting fluid were plated in duplicate on both tryptone agar (pH 7.0) and M-9 agar, incubated for 24 and $48 \mathrm{hr}$., respectively, at $37^{\circ}$ and counted. All manipulations subsequent to ultraviolet irradiation were carried out in subdued light to minimize photoreactivation. Sensitivity to ultraviolet radiation was calculated as ergs $/ \mathrm{mm}^{-2} / \mathrm{hit}$, a hit being defined as - ln (fraction of survivors).

\section{RESULTS}

First-step mutants. The results of a survey of 100 potential first-step mutants resistant to Mitomycin $\mathrm{C}$ are presented in Table 2 . The cross-resistance patterns of the various types of mutants are given in Table 3.

Only four of the 100 isolates were indistinguishable from the parent strain S. The remainder showed some degree of resistance to Mitomycin $\mathrm{C}$. These could be divided into two main groups: those which were cross-resistant with all radiomimetic compounds (radioresistant) and those which were not (chemoresistant). The latter group could be subdivided into two subgroups. One, the prototype of 
Table 2. Classification of survivors after treating Escherichia coli S with Mitomycin $C$

\begin{tabular}{|c|c|c|c|c|c|c|c|c|c|c|c|c|}
\hline \multirow{3}{*}{$\begin{array}{c}\text { Mytomycin } \\
\text { C } \\
(\mu \mathrm{g} . / \mathrm{ml} .)\end{array}$} & \multirow{3}{*}{$\begin{array}{l}\text { Surviving } \\
\text { colonies } \\
\text { (18 hr.) }\end{array}$} & \multirow{3}{*}{$\begin{array}{l}\text { No. } \\
\text { tested }\end{array}$} & \multirow{3}{*}{$\begin{array}{l}\text { Sensi- } \\
\text { tive }\end{array}$} & \multicolumn{9}{|c|}{ No. of type } \\
\hline & & & & \multicolumn{7}{|c|}{ Radioresistant } & \multicolumn{2}{|c|}{$\begin{array}{l}\text { Chemo- } \\
\text { resistant }\end{array}$} \\
\hline & & & & $\mathbf{R}_{1}$ & $\mathbf{R}_{\mathbf{2}}$ & $\mathbf{R}_{3}{ }^{*}$ & $\mathbf{R}_{4}{ }^{*}$ & $\mathbf{R}_{5}^{*}$ & $\mathbf{R}_{6}{ }^{*}$ & $\mathbf{R}_{7} *$ & $\mathbf{I}$ & II \\
\hline 0.03 & $\begin{array}{l}\text { Confluent } \\
\text { growth }\end{array}$ & & & & & & & & & & & \\
\hline 0.08 & $300-400$ & 25 & 4 & 0 & $\mathbf{0}$ & 8 & 1 & $\mathbf{0}$ & 1 & $\mathbf{0}$ & 7 & 4 \\
\hline $0 \cdot 15$ & 200 & 25 & $\mathbf{0}$ & 0 & $\mathbf{0}$ & 13 & 11 & 1 & $\mathbf{0}$ & $\mathbf{0}$ & 0 & $\mathbf{0}$ \\
\hline $\mathbf{0 \cdot 3 0}$ & 60 & $\mathbf{3 0}$ & $\mathbf{0}$ & $\mathbf{0}$ & 0 & 13 & 15 & 2 & $\mathbf{0}$ & $\mathbf{0}$ & $\mathbf{0}$ & $\mathbf{0}$ \\
\hline $\mathbf{0 . 5 0}$ & 15 & 9 & $\mathbf{0}$ & $\mathbf{0}$ & $\mathbf{0}$ & $\mathbf{0}$ & 8 & $\mathbf{0}$ & $\mathbf{0}$ & 1 & $\mathbf{0}$ & $\mathbf{0}$ \\
\hline 0.75 & $5(8 \dagger)$ & 8 & $\mathbf{0}$ & $\mathbf{0}$ & $\mathbf{0}$ & $\mathbf{0}$ & 8 & $\mathbf{0}$ & $\mathbf{0}$ & $\mathbf{0}$ & $\mathbf{0}$ & 0 \\
\hline $1 \cdot 0$ & $1(3 \dagger)$ & 3 & $\mathbf{0}$ & $\mathbf{0}$ & $\mathbf{0}$ & $\mathbf{0}$ & $\mathbf{3}$ & $\mathbf{0}$ & $\mathbf{0}$ & $\mathbf{0}$ & $\mathbf{0}$ & $\mathbf{0}$ \\
\hline $\mathbf{8} \cdot \mathbf{0}$ & $\mathbf{0}$ & - & - & - & - & - & - & - & - & - & - & - \\
\hline Totals & & 100 & 4 & 0 & $\mathbf{0}$ & 34 & 46 & $\mathbf{3}$ & 1 & 1 & 7 & 4 \\
\hline
\end{tabular}

* Cross-resistant pattern of representative strains of each type S/Mc $1 \mathrm{~g}, \mathrm{~S} / \mathrm{Mc} 1 \mathrm{~b}, \mathrm{~S} / \mathrm{Mc} 1 \mathrm{f}$, S/Mc 1 i, S/Mc $1 \mathrm{~h}, \mathrm{~S} / \mathrm{Mc} 1 \mathrm{c}$, and S/Mc $1 \mathrm{~d}$, respectively, are shown on Table 1.

+ After $42 \mathrm{hr}$.

which is S/Mc 1 c, was resistant (2.5-fold) only to Mitomycin C and is therefore clearly chemoresistant. The other subgroup of which $S / M c 1 d$ is representative, was cross-resistant with nitrosoguanidines and penicillin but not with any of the other chemical agents. The response of S/Mc $1 \mathrm{~d}$ and $\mathrm{S}$ to ultraviolet radiation (Fig. I) was identical when both were plated on tryptone agar subsequent to irradiation. When, however, both were plated on M-9 agar, the slope of the survival curve of S increased six-fold, but that of S/Mc $1 \mathrm{~d}$ increased 10- to 12-fold. This is the first instance in which the difference between radio- and chemoresistance is not clearcut. For the present, mutants of this group have been classified as chemoresistant, with the recognition that this is an arbitrary classification of an anomalous strain.

Table 3. Cross-resistance relationships among first-step Mitomycin C resistant mutants of Escherichia coli strain $S$ relative to the sensitive parent strain

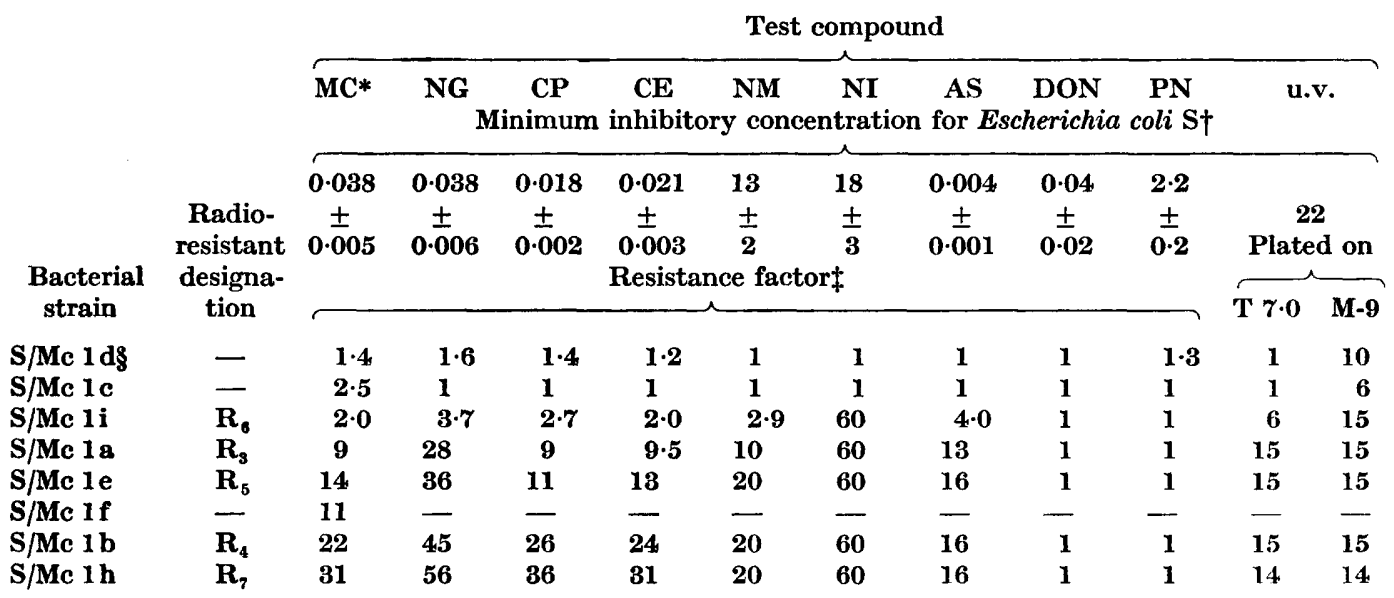

$*, \uparrow, \ddagger$, \&. See footnotes to Table 1 . 


\section{J. Greenberg, J. D. Mandell and P. L. Woody}

Five distinct types of radioresistant mutants were isolated. $54 \%$ of the radioresistant mutants, represented by $S / M c 1 b$, had a cross-resistance pattern identical with that of all previously isolated mutants of type $R_{\mathbf{4}} ; \mathbf{4 0} \%$, represented by $\mathrm{S} / \mathrm{Mc} \mathbf{1} \mathrm{g}$, were indistinguishable from other members of type $\mathbf{R}_{3}$. The remaining five radioresistant mutants had cross-resistance patterns not previously observed. $\mathrm{S} / \mathrm{Mc} \mathrm{le}, \mathrm{S} / \mathrm{Mc}$ If and $\mathrm{S} / \mathrm{Mc} \mathrm{Ih}$ were identical to $\mathrm{R}_{4}$ in their degree of resistance to ultraviolet radiation, nitrogen mustard, nitromin and azaserine, and in their lack of resistance to DON and penicillin. However, S/Mc $1 \mathrm{e}$ and S/Mc 1 f were somewhat less resistant than $R_{4}$ to Mitomycin $\mathrm{C}$ and the nitrosoguanidines, and $\mathrm{S} / \mathrm{Mc} \mathbf{1 h}$ was

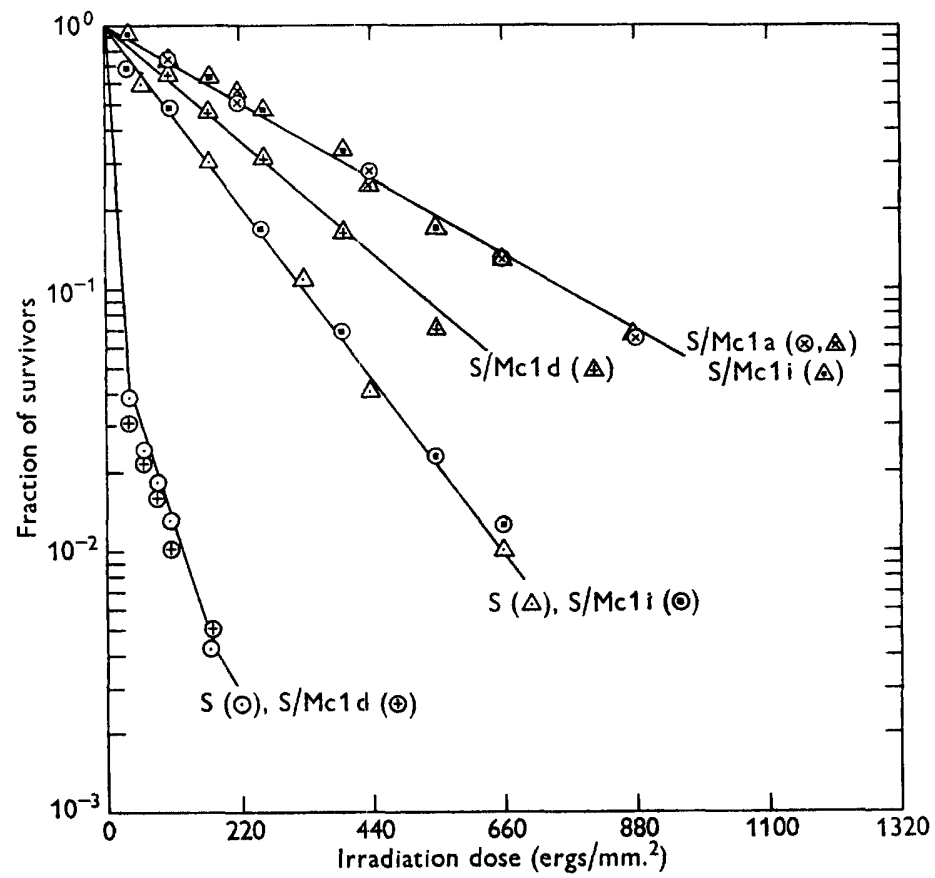

Fig. 1. Survival of Escherichia coli S, chemoresistant strain S/Mc 1d, and radioresistant strains $\mathrm{S} / \mathrm{Mc} \mathrm{I}$ a and $\mathrm{S} / \mathrm{Mc} \mathrm{I} i$ exposed to ultraviolet radiation. $O$, Survivors plated on tryptone agar, pH 7•0. $\triangle$, survivors plated on M-9 defined medium, pH 6.8.

somewhat more resistant than $R_{4}$ to these same compounds. S/Mc 1e and S/Mc If differed from each other only in a small, but seemingly consistent, manner on repeated trials, with regard to their resistance to Mitomycin $\mathrm{C}$. They have been arbitrarily classed together as $\mathbf{R}_{\mathbf{5}}$. S/Mc $1 \mathrm{~h}$ has been designated $\mathbf{R}_{\mathbf{7}}$.

The last, hitherto undescribed, radioresistant mutant, S/Mc 1i, had a crossresistance pattern very similar to that of $\mathbf{R}_{1}$. However, unlike $\mathbf{R}_{1}$, which was 15 -fold resistant to ultraviolet radiation irrespective of post-irradiation plating medium, S/Mc 1 i was six-fold resistant when plated on complex (tryptone) medium and 15-fold resistant when plated on M-9 agar (Fig. 1). S/Mc $1 \mathrm{i}$ is, therefore, considered different from $R_{1}$ and has been designated $R_{6}$.

The frequency distributions shown in Table 2 should be interpreted with caution. Nevertheless certain facts can be derived from the table. The frequency with which 
a type of mutant was selected depended to some extent on the concentration of the drug. The mutants with the lowest degree of resistance to Mitomycin $\mathrm{C}$ were selected only from the plate with the lowest concentration of Mitomycin $\mathrm{C}$ at which there was not confluent growth. This plate was the only one from which parental, sensitive organisms were picked. As the concentration of Mitomycin $\mathrm{C}$ was increased, only the more resistant mutants were isolated. It would appear from the sampling of survivors over a 40 -fold range of concentration of Mitomycin $\mathrm{C}$ that radioresistant mutants predominated and that most of these were $\mathbf{R}_{3}$ or $\mathbf{R}_{4}$. However, half the mutant resistant population sampled from the plate containing Mitomycin $\mathbf{C}$ at $0.08 \mu \mathrm{g}$. $/ \mathrm{ml}$. were chemoresistant. It is curious that from this same plate only one $\mathbf{R}_{\mathbf{4}^{-}}$ type mutant was isolated. Assuming no sampling prejudice, this would mean that there were $16 \mathrm{R}_{4}$ mutants on the plate, whereas at the next higher concentration of Mitomycin $\mathrm{C}$ there were $\mathbf{8 8}$ and the frequency decreased thereafter as the concentration of Mitomycin C increased. Actually the sampling of the $0.08 \mu \mathrm{g} . / \mathrm{ml}$. plate was probably prejudiced, since there were several colony sizes and an almost equal number of each colony size was picked regardless of the true frequency of the various colony sizes on the plate. Only the mutants of the type S/Mc $1 \mathrm{~d}$ and sensitives were found among the smallest colony type.

Table 4. Cross-resistance relationships among mutants of Escherichia coli $\boldsymbol{S}$ representing five consecutive steps in resistance to Mitomycin C

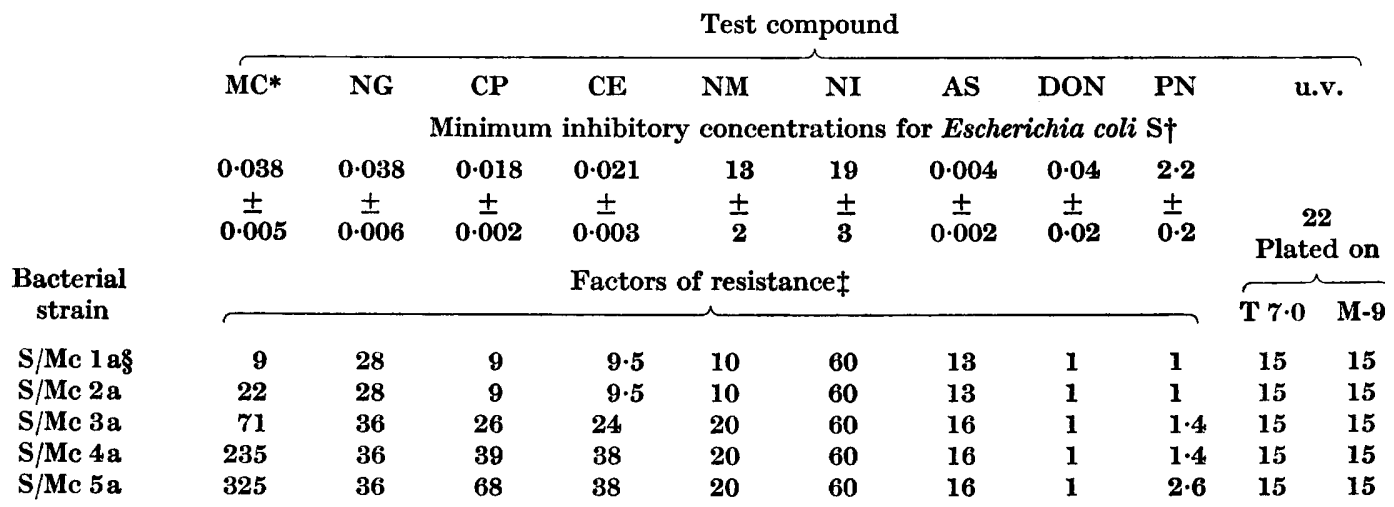

$*, \uparrow, \ddagger$, §. See footnotes to Table 1 .

Granting that the frequency of occurrence of any mutant type depends on the selective pressure of the Mitomycin C, nevertheless mutants of the types $\mathbf{R}_{5}, \mathbf{R}_{6}$ and $\mathbf{R}_{7}$ were rare compared to those of types $\mathbf{R}_{\mathbf{3}}$ and $\mathbf{R}_{\mathbf{4}}$.

Sequential steps in resistance to Mitomycin C. Mutants representing five sequential steps in increasing resistance to Mitomycin $\mathrm{C}$ were selected as follows: $\mathrm{S} / \mathrm{Mc} 1$ a was selected from S, S/Mc 2 a from S/Mc 1a, S/Mc 3a from S/Mc 2a, etc., as seen in Table 4. The second step mutant, $\mathrm{S} / \mathrm{Mc} 2 \mathrm{a}$, was more resistant than its parent only to Mitomycin C. The third-step mutant, $\mathbf{S} / \mathbf{M c ~ 3 a}$, increased in resistance to all radiomimetic agents. Its cross-resistance pattern was essentially that of an $\mathbf{R}_{\mathbf{4}}$ radioresistant mutant except that $\mathbf{S} / \mathbf{M c} \mathbf{3}$ a was more resistant to Mitomycin $\mathrm{C}$ than $\mathbf{R}_{\mathbf{4}}$ mutants. The fourth-step mutant increased in resistance not only to Mitomycin $\mathbf{C}$ 
but also to 1-chloropropyl- and 1-chloroethyl-3-nitro-1-nitrosoguanidine. The fifthstep mutant increased in resistance to Mitomycin $\mathrm{C}$, to 1-chloropropyl-3-nitro1-nitrosoguanidine and to penicillin.

The survival curves of the second- to fifth-step mutants in Mitomycin C resistance, as well as those of $R_{1}, R_{2}, R_{4}, R_{5}$, and $R_{7}$ after exposure to ultraviolet radiation were indistinguishable from that of $S / M c 1 a$ (Fig. 1) and were not altered by post-irradiation growth in complex or synthetic medium.

\section{DISCUSSION}

The orginal purpose of the work described here was to determine whether, by cross-resistance studies, relationships could be established among compounds which for various reasons (Mandell et al. 1961) were considered to be radiomimetic. It has been shown in this paper and previous papers of this series that there exists a class of compounds such that mutants of Escherichia coli $\mathrm{S}$ selected for resistance to one member of the class will be resistant to all members of the class and to ultraviolet radiation. Most of the mutants of $\boldsymbol{E}$. coli $\mathrm{S}$ selected for resistance to 1-methyl3-nitro-1-nitrosoguanidine (Mandell et al. 1961), nitrogen mustard and nitromin (Woody et al. 1961), azaserine and other nitrosoguanidines (Greenberg et al. 1961) and, as shown in this paper, Mitomycin $\mathrm{C}$, were also resistant to ultraviolet radiation and to each of the other radiomimetic agents tested. This cross-resistance was not the result of a general increase in resistance to all antibacterial agents, for none of the radioresistant mutants of $\mathrm{S}$ was significantly resistant to 6-diazo-5-oxo-Lnorleucine or penicillin. The observations regarding mutual cross-resistance among radiomimetic agents and radiation are extensions of earlier observations of Bryson (1948) who found that Witkin's (1947) strains of $\boldsymbol{E}$. coli B, selected for resistance to radiation, were also resistant to nitrogen and sulphur mustard. Conversely, strains selected for resistance to nitrogen mustard were resistant to radiation (Bryson, 1948). Furthermore, the radiation-resistant mutant of $E$. coli $B$, strain $B / r$, was similarly found resistant to nitrofuracin, and strains selected for resistance to nitrofuracin were also resistant to radiation (Szybalski \& Nelson, 1954).

However, not all mutants selected for resistance to radiomimetic compounds are radioresistant. It was shown earlier that one mutant selected for resistance to nitromin was resistant only to nitromin and not to other radiomimetic agents-not even the chemically similar nitrogen mustard-nor to radiation (Woody et al. 1961). It has been shown in this paper that there is at least one first-step mutant resistant to Mitomycin C (S/Mc 1c) which is resistant only to Mitomycin C. There was another mutant which could not so unequivocally be classified as chemoresistant. S/Mc Id, which appeared as sensitive as the parent $\mathbf{S}$ to ultraviolet radiation when plated on complex medium, was reactivated to a much greater extent than $S$ when the organisms were plated on M-9 agar. Furthermore, S/Mc $1 \mathrm{~d}$ was resistant not only to Mitomycin $\mathrm{C}$ but was also resistant to the nitrosoguanidines and to penicillin.

There are other examples where resistance to Mitomycin $\mathrm{C}$ and one or more nitrosoguanidines increase or decrease together without any concomitant change in resistance to any of the other agents used in these studies. Mutants classified as $\mathbf{R}_{5}$ and $R_{7}$ (Table 3 ) have cross-resistance patterns identical with $R_{4}$ except for their resistance to Mitomycin $\mathbf{C}$ and the nitrosoguanidines, $\mathbf{R}_{5}$ being somewhat less and 
$\mathbf{R}_{\mathbf{7}}$ somewhat more resistant to all these agents than $\mathbf{R}_{4}$. S/Mc $4 \mathbf{a}$ and 5 a (Table 4) were characterized by increases in resistance only to Mitomycin $\mathrm{C}$ and to one or more nitrosoguanidines; $\mathrm{S} / \mathrm{Ng} 2 \mathrm{a}$, a second-step mutant selected for resistance to 1-methyl-3-nitro-1-nitrosoguanidine displayed an increase in resistance to Mitomycin $\mathrm{C}$ (Mandell et al. 1961). These data suggest that Mitomycin $\mathrm{C}$ and the nitrosoguanidines may have in common a chemically reactive group or a mode of action, not necessarily radiomimetic. The elucidation of the chemical structure of Mitomycin $\mathrm{C}$ will tell whether the former possibility is true.

Seven different first-step radioresistant mutants have been isolated to date from the parent strain $S$. Two of these, $R_{3}$ and $R_{4}$, have been isolated repeatedly using a variety of different radiomimetic agents for selection. $\mathbf{R}_{3}$ - and $\mathbf{R}_{4}$-types comprised the overwhelming majority of the radioresistant mutants isolated when Mitomycin $\mathbf{C}$ was the selecting agent (Table 2). The other five radioresistant types were rare, $\mathbf{R}_{\mathbf{1}}$, $\mathbf{R}_{2}, \mathbf{R}_{6}$ and $\mathbf{R}_{7}$ having been selected once each from among mutants resistant to each of five different radiomimetic agents. Three mutants of the type $\mathbf{R}_{5}$ were isolated when Mitomycin $\mathbf{C}$ was the selecting agent but not when any other radiomimetic agent was used. It should be pointed out, however, that the current survey of 100 potential resistant mutants is the most extensive we have undertaken. It is, therefore, impossible to decide whether $R_{1}$ was induced more frequently by 1 -chloropropyl-3-nitro-1-nitrosoguanidine, $R_{2}$ by nitromin, and $R_{5}, R_{6}$ and $R_{7}$ by Mitomycin $\mathrm{C}$, or whether, being rare, they were selected by these particular agents by chance. It is also difficult to decide whether the rare $\mathrm{R}$-types are actually the result of single mutational events, or whether they represent double mutations. $\mathbf{R}_{\mathbf{5}}$ and $\mathbf{R}_{\mathbf{7}}$ might conceivably be $R_{3}$ and $R_{4}$, respectively, with a second, chemoresistant mutation to Mitomycin $C$ resistance. $R_{1}$ and $R_{2}$, having such a low degree of resistance to all radiomimetic compounds, would have to be $R_{3}$ or $R_{4}$, modified by a second mutation to a lower degree of resistance to all radiomimetic compounds. $\mathbf{R}_{6}$, differing as it does from all other $\mathbf{R}$-types in its plating medium reactivation characteristics, is probably the product of a single mutation. Based on preliminary estimates of the frequency with which the presumed primary mutations to resistance occur $\left(c 3 \times 10^{-6}\right.$ for $R_{3}$ and $R_{4}$ and the chemoresistant type of $S / M c 1 c$ with Mitomycin $C$ at 0.08 $0.15 \mu \mathrm{g} . / \mathrm{ml}$.) it seems unlikely that double mutations would be selected from among the relatively small samples plated ( $3 \times 10^{7}$ on each of the Mitomycin C plates).

All the radioresistant mutants of Escherichia coli $\mathrm{S}$ differ in several ways from the radioresistant mutant of $E$. coli $\mathrm{B}, \mathrm{B} / \mathrm{r}$ (Witkin, 1947). The degree of resistance to ultraviolet radiation of all radioresistant types of $S$, except $\mathbf{R}_{6}$, was unmodified by the type of medium on which they were plated following irradiation. $E$. coli $\mathrm{B} / \mathrm{r}$, on the other hand, appears to be more resistant than strain $B$ to ultraviolet radiation only when both strains are plated on complex medium (Alper \& Gillies, 1960). When both $B$ and $B / r$ were plated on defined medium following irradiation, they had indistinguishable survival curves. This is because $B$ is more sensitive to irradiation when plated on complex medium than when plated on defined medium (Roberts \& Aldous, 1949), while the opposite is true of $\mathrm{B} / \mathrm{r}$. E. coli $\mathrm{S}$ behaves with respect to plating medium reactivation like $E$. coli $B$; the radioresistant type $\mathbf{R}_{6}$ behaves, qualitatively, like $E$. coli $\mathrm{B}$ and $\mathrm{S}$, the opposite to $\mathrm{B} / \mathrm{r}$, and differently from all the other R-types of $S$.

$\mathrm{B} / \mathrm{r}$ also differs from the $\mathrm{R}$-types of Escherichia coli $\mathrm{S}$ with respect to collateral 
resistance to penicillin and the sulphonamides. According to Witkin (1947) and Adler \& Haskins (1960), B/r is resistant to penicillin and to sulphathiazole as well as to radiation. None of the $R$-types of $S$ was resistant to penicillin and none of those tested $\left(R_{1}\right.$ to $R_{4}$ ) was resistant to sulphonamides (unpublished). Moreover, while $B / r, R_{3}$ and $R_{4}$ do not, as do $B$ and $S$, respond to the challenge of radiation by forming filaments, filament formation after irradiation is characteristic of $\mathbf{R}_{\mathbf{1}}$ and $\mathbf{R}_{\mathbf{2}}$ (Curry \& Greenberg, unpublished).

Assuming all the $\mathbf{R}$-types of strains $\mathbf{S}$ are each due to a single mutation, there may be as many as $\mathbf{1 7}$ mutations involved in the resistance or sensitivity of Escherichia coli to radiation. Witkin (1947) recognized four different groups of radiationresistant mutants of $E$. coli $B$. One group, represented by $B / r$, was resistant to penicillin and sulphathiazole; another was resistant to penicillin but not sulphathiazole; another was resistant to sulphathiazole but not to penicillin; and a fourth group was resistant to neither of these agents. Two of these mutants, the two with concomitant resistance to penicillin, are obviously different from any of the R-types of $\mathrm{S}$. The other two radioresistant mutants of $\mathrm{B}$ cannot yet be clearly distinguished from all the $\mathbf{R}$-types of $\mathrm{S}$. Also not yet fully distinguishable from the R-types of $\mathbf{S}$ are the radiation sensitive mutants of $B$, described by Hill \& Simson (1961) although $B_{\mathrm{S}}$ and $B_{\mathrm{s}-2}$ appear to be more sensitive than $S$. With respect to radiation sensitivity together with collateral resistance to chemical agents, $E$. coli $\mathrm{B}$ exists in at least seven forms, E. coli $\mathrm{S}$ in eight. All the forms of $\mathrm{B}$ might be counterparts of those in $\mathrm{S}$, differing only in one mutation from $B$ to $S$, or vice versa. If this were the case, there would be nine mutations controlling radiation sensitivity and collateral resistance to other agents in $E$. coli. If, on the other hand, none of the mutants of $B$ were counterparts of any of those in $S$, and $B$ and $S$ were different, there might be as many as 16 mutational events controlling radioresistance to $E$. coli. Furthermore, B/r would appear to be about as resistant to radiation as the $\mathrm{R}$-types of $\mathrm{S}$, but $\boldsymbol{E}$. coli $\mathbf{H}$, is, according to our experience, about twice as resistant to ultraviolet radiation as the R-types of S. Assuming, then, that a single mutation controls the difference between $\mathrm{S}$ and $\mathrm{H}$, with respect to radiation sensitivity, there may be as many as 17 mutational events controlling radiation sensitivity in $E$. coli. By integrating the published data with our own experience we would estimate that the difference between the most radiation sensitive and resistant forms of $E$. $\operatorname{coli}\left(\mathrm{B}_{\mathrm{s}}\right.$ of Hill \& Simson, 1961, and $\mathrm{H}$ ) in resistance to ultraviolet radiation to be about 200 -fold.

The isolation of five consecutive steps in resistance to Mitomycin $\mathrm{C}$ appears to reflect the interaction between radio- and chemoresistant mutations. S/Mc 2a may be considered to consist of an $R_{3}$ plus a chemoresistant mutation similar to or identical with S/Mc I c. The third-step mutant, S/Mc 3a, probably represents a shift from $\mathbf{R}_{3}$ to $\mathbf{R}_{4}$, superimposed on its chemoresistant mutation acquired in the previous step. Previously it was clear that no R-group was a prerequisite for any other R-group; that is, all the R-groups could be selected in one step from the parent sensitive strain. Furthermore, in earlier experiences with nitrosoguanidines, nitromin or nitrogen mustard no R-group was selected from any other R-group. It was as though all $\mathbf{R}$-types were mutually exclusive. Now it is evident from the change in resistance patterns from S/Mc 2 a to S/Mc 3 a that mutations can occur from one $\mathbf{R}$ group to another. Such a shift would appear to require a single genetic change. S/Mc 4, a and S/Mc 5a, in turn, may represent either the imposition of additional 
chemoresistant steps on a basic $\mathbf{R}_{\mathbf{4}}$ radioresistance or a change from $\mathbf{R}_{\mathbf{4}}$ to $\mathbf{R}_{\mathbf{7}}$. It should be noted that as many as three of the five steps in resistance to Mitomycin $\mathrm{C}$ may represent chemoresistant mutations, but only two such mutations were identified as first-step mutants. This might mean that certain chemoresistant mutations can occur only after another one has occurred; or that among the first-step chemoresistant mutants there are two phenotypes for three genotypes; or that one chemoresistant mutation has not yet been discovered; or, as suggested above, one of the steps is a change from $\mathbf{R}_{4}$ to $\mathbf{R}_{\mathbf{7}}$.

It is evident from this discussion that there are many different radioresistant mutants of Escherichia coli, each with a characteristic cross-resistance pattern to ultraviolet radiation, a variety of radiomimetic and some non-radiomimetic agents. Genetic analyses of these mutants are planned, for without such an analysis it is difficult to determine whether the mutants represent different alleles at one locus or mutations at different loci. Information of this nature will be a useful guide for postulating models to account for resistance to the lethal effects of radiation and radiomimetic chemicals.

This work was carried out under the auspices of the Cancer Chemotherapy National Service Center, National Cancer Institute, U.S. Public Health Service, Contract No. SA-43-ph-3070 and Grant No. CY-4548 and Cy-5687.

\section{REFERENCES}

AdLer, H. I. \& Haskins, S. D. (1960). Heterogeneity of cultures of Escherichia coli B/r. Nature, Lond. 188, 249.

AlPER, T. \& Gillies, N. E. (1960). The relationship between growth and survival after irradiation of Escherichia coli strain B and two resistant mutants. J. gen. Microbiol. 22, 113.

Bryson, V. (1948). The effects of nitrogen mustard on Escherichia coli. J. Bact. 56, 423.

Greenberg, J., Mandell, J. D. \& Woody, P. L. (1961). Resistance and cross-resistance of Escherichia coli mutants to radiomimetic agents. A preliminary report. Cancer Chemotherapy Reports, no. 11, p. 51.

Hill, R. F. \& Srmson, E. (1961). A study of radiosensitive and radioresistant mutants of Escherichia coli strain B. J. gen. Microbiol. 24, 1.

Latarjet, R., Morenne, P. \& Berger, R. (1953). Un appareil simple pour le dosage des rayonnements ultraviolets emis par les lampes germicides. Ann. Inst. Pasteur, 85, 174.

Mandel., J. D., Woody, P. L. \& GreenberG, J. (1961). Resistance and cross-resistance of Escherichia coli mutants to anticancer agents. 1-methyl-3-nitro-1-nitrosoguanidine. J. Bact. 81, 419.

Овознг, S. (1959). Cross-resistance between Mitomycin $\mathrm{C}$ and alkylating agents in experimental cancer chemotherapy. Gann, 50, 147.

Roberts, R. B. \& Aldous, E. (1949). Recovery from ultraviolet irradiation in Escherichia coli. J. Bact. 57, 363.

Shiba, S., Terawaki, A., Taguchi, T. \& Kawamata, J. (1959). Selective inhibition of formation of deoxyribonucleic acid in Escherichia coli by Mitomycin C. Nature, Lond. $183,1056$.

SzYBALSKI, W. (1958). Special microbiological systems. II. Observations on chemical mutagenesis in micro-organisms. Ann. N.Y. Acad. Sci. 76, 475.

SzyBalski, W. \& Bryson, V. (1952). Genetic studies on microbial cross-resistance to toxic agents. I. Cross-resistance of Escherichia coli to fifteen antibiotics. J. Bact. 64, 489.

Sybalski, W. \& Nelson, T. C. (1954). Genetics of bacterial resistance to nitrofurans and radiation. Bact. Proc. 1954, 51. 
Usubuchi, I., Oboshi, S., Tsuchida, R., Tazawa, S., Narita, N., Tanabe, H., Takoshi, F., Matusumoto, T. \& Narita, K. (1957). Inhibitory effect of Mitomycin $\mathrm{C}$ upon experimental tumors. Gann, 48, 447.

WITKIN, E. (1947). Genetics of resistance to radiation in Escherichia coli. Genetics, $32,221$.

Woody, P. L., Mandell, J. D. \& Greenberg, J. (1961). Resistance and cross-resistance of Escherichia coli mutants to anticancer agents. Nitrogen mustard and nitromin. Radiat. Res. (in the Press). 